\title{
Stem cell secretome, regeneration, and clinical translation: a narrative review
}

\author{
Chukwuweike Gwam, Nequesha Mohammed, Xue Ma
}

Department of Orthopedic Surgery, Wake Forest School of Medicine, Wake Forest Baptist Medical Center, Winston-Salem, NC, USA

Contributions: (I) Conception and design: All authors; (II) Administrative support: C Gwam, X Ma; (III) Provision of study materials or patients: All authors; (IV) Collection and assembly of data: C Gwam, N Mohammed; (V) Data analysis and interpretation: C Gwam, N Mohammed; (VI) Manuscript writing: All authors; (VII) Final approval of manuscript: All authors.

Correspondence to: Chukwuweike Gwam, MD, MBA. Department of Orthopedic Surgery, Wake Forest School of Medicine, Wake Forest Baptist Medical Center, Medical Center Boulevard, Winston-Salem, NC 27157, USA. Email: cgwam1988@gmail.com.

\begin{abstract}
Regenerative medicine is a field growing in popularity due to high hopes for stimulating in situ tissue restoration. Stem cell therapy remain at the center of regenerative medicine, due to early reports on its pluripotent differentiating capability. However, more recent reports suggest the paracrine activity of stem cells, and not direct differentiation, as the cause of its therapeutic effects. This paracrine activity can be harnessed in the form of conditioned media. Despite these capabilities, the clinical translation of stem cell conditioned media (i.e., secretome) is precluded by a variety of factors. These limitations include standardization of stem cell-conditioned media formulation, characterization of bioactive factors in conditioned media and dosing, optimizing modes of delivery, and uncovering of mechanisms of action of stem cell conditioned media. The purpose of this review is to provide a focused narration on the aforementioned preclusions pertaining to the clinical translation of stem cell conditioned media. Specifically, we will report on commonly use methodologies for the development of stem cell conditioned media, modalities for conditioned media characterization, modes of delivery, and postulated mechanisms of action for stem cell conditioned media in regenerative medicine.
\end{abstract}

Keywords: Regenerative medicine; stem cell therapy; stem cell conditioned media; stem cell secretome

Submitted Jul 29, 2020. Accepted for publication Dec 01, 2020.

doi: $10.21037 / \mathrm{atm}-20-5030$

View this article at: http://dx.doi.org/10.21037/atm-20-5030

\section{Introduction}

Mammals and humans have a limited ability for the self-regeneration of tissue and organs. Some tissues systems, such as long bone and the human liver, harbor prolific regenerative capacity. However, other critical organs such as the heart and central nervous system, often employ an innate repair process specific to the site of injury. Unfortunately, innate repair processes are less than adequate and render a less functional organ system than the pre-diseased state. For example, the consequence of chondral lesions is often fibrocartilage replacement followed by joint space narrowing and bony outgrowth into an articular space. Similarly, damage to cardiac tissue and the central nervous system stimulates fibrotic replacement of the tissue and a reduction in functionality of the organ $(1,2)$. Even tissues with significant regenerative potential, such as the peripheral nerve, skeletal muscle, and liver, can witness a significant reduction in regenerative capabilities when confronted with specific factors. These factors include age, persistent oxidative stress, chronicity of infliction, and various comorbid states (3).

Regenerative medicine is an interdisciplinary field that applies medicine, science, and engineering principles to enhance the intrinsic regenerative capacity of the host. One method in particular that has received an increased amount of attention has been the application of stem-cell based therapies $(4,5)$. Stem cells are non-specialized cells with high differentiation and self-renewal potential (6). 
On the basis of regenerative applications, stem cells can be categorized as mesenchymal stem cells (MSCs), tissue specific progenitor stem cells (TSPCs), bone marrow stem cells (BMSCs), induced pluripotent stem cells (IPSCs), and embryonic stem cells (ESCs) (7). MSCs and BMSCs are most often harvested from adipose tissue and bone marrow respectively and harbor high multipotent potential. Tissue specific progenitor cells have limited differentiation potential, and can differentiate to cell types specific to the tissue from which they are acquired (8). Alternatively, IPSCs are derived from adult somatic cells and are reprogrammed into a pluripotent state by induced expression of certain transcription factors (9-12). Embryonic stems cells are pluripotent and may confer an advantage over MSCs, BMSCs and TSPCs. Furthermore, ESCs can be harvested and grown relatively easily and for longer durations in cell culture (13).

Certain factors are necessary for the in vivo regenerative success of stem-cell based therapies. These factors include adequate migration of the stem cell to the site of injury, stem cell engraftment and differentiation at the site of injury, and stem cell survival $(6,14)$. However, risks such as teratoma formation and immune rejection, persist even after proper engraftment of stem cells (15). These limitations have severely handicapped the in vivo use of stem cells for tissue regeneration (16). Fortunately, stem cell conditioned media, or alternatively named secretome and/or exosome, has been described as an effective alternative. This conclusion was surmised by Gnecchi et al., who revealed that MSCs mediated their therapeutic effects by the secretion and release of trophic molecules known as secretomes (17). Further work by Teixeira $e t a l$. provided more evidence that suggested that the secretome is the primary cause of stem cell mediated repair and regeneration (18). As such, there has been studies devoted to exploring the therapeutic potential of stem cell secretome in conditioned media. While promising, certain limitations persist that preclude the widespread implementation of stem cell conditioned media. These limitations include standardization of stem cell-conditioned media (i.e., secretome) formulation, characterization of bioactive factors in conditioned media and dosing, optimizing modes of delivery, and uncovering of mechanisms of action of stem cell conditioned media. The purpose of this review is to provide a focused narration on the aforementioned limitations pertaining to the clinical translation of stem cell conditioned media. Specifically, we will report on commonly use methodologies for the development of stem cell conditioned media, modalities for conditioned media characterization, modes of delivery, and postulated mechanisms of action for stem cell conditioned media in regenerative medicine. We present the following article in accordance with the Narrative Review reporting checklist (available at http://dx.doi.org/10.21037/atm-20-5030).

\section{Methods}

An electronic systematic review of the literature was conducted using the Medline Database. MEDLINE is the U.S. National Library of Medicine ${ }^{\circledR}$ (NLM) premier bibliographic database that contains more than 26 million references to journal articles in life sciences with a concentration on biomedicine. A distinctive feature of MEDLINE is that the records are indexed with NLM Medical Subject Headings $\left(\mathrm{MeSH}^{\circledR}\right)$. MEDLINE is the online counterpart to MEDical Literature Analysis and Retrieval System (MEDLARS ${ }^{\circledR}$ ) that originated in 1964 (19). Studies of interest were identified using the following keyword combinations and Boolean operators: "stem cell" AND "conditioned media"; "stem cell" AND "secretome"; "stem cell" AND "exosome" and were limited for studies published within 10 years of the date of the search. Our search was performed in April 2020. Exclusion criteria included manuscripts not written in English, conference reviews, editorials, surveys, case reports, and case series. This included $~ 3,000$ articles were further screened for relevance to the topic of the present review.

\section{Discussion}

\section{Formulation of conditioned media}

Several methodologies exist for the formulation of stem cell conditioned media. However, they all converge on the following methodology: (I) harvesting and plating of stem cells on tissue culture plate until $60-80 \%$ confluency (II) extraction of supernatant media (conditioned media); and (III) centrifugation/lyophilization and/or precipitation by ultrafiltration or trichloroacetic acid of conditioned media and storage (20). Harvesting and plating methods are of critical importance as it serves as foundation of secretome efficacy. Researchers should adhere to well established methodologies specific to harvesting and plating stem cells. Additionally, phenotypic analyses, such as flow cytometry are critical to ensure that cell line used have not undergone significant genetic drift so much so that experimental results become irreproducible $(21,22)$. Care must be taken to limit 
contaminants from basal cell media among the supernatant collection. Standard filtration methods utilize $0.22 \mu \mathrm{M}$ pores with varying centrifugation speeds that are often cell line specific. Proteomic analysis may allow the researcher the ability to identify the percentage of basal cell media contaminant in the secretome.

Significant variability exists in the literature at critical points of conditioned media formulation. These variations are many. As such, we will limit to the most common variations that arose in the literature. These include type of basal culture media, oxidative conditions evoked on the cell culture, dimensionality of cell culture, and the use of fetal bovine serum (FBS). Basal culture media vary significantly in studies utilizing stem cell conditioned media. These include alpha-MEM media, DMEM, DMEM/F12, M199, EBM2, EGM-2, and ex vivo 15 (23). Sagaradze et al. (24) reported varying concentrations of cytokines and proteins among human MSC secretome cultured in either low glucose DMEM or proprietary NutriStem medium (Biological Industries, Israel). To our knowledge, there are no studies that directly compares the effect of various basal media on therapeutic effectiveness of stem cell secretomes.

Oxygen tension in which stem cells are cultured, vary from normoxic culture conditions $\left(21 \%, \mathrm{O}_{2}\right)$ to variable oxygen deprived conditioned (hypoxia $\mathrm{O}_{2}$ level $0.5 \%$ to $2 \%$ ). These variations have significant effects on the biomolecular profile of stem cell conditioned media. For example, some studies report that growth factors such as platelet derived growth factor, hepatocyte growth factor, placenta growth factor, and vascular endothelial derived growth factor, are upregulated in hypoxic conditions (25-28). This upregulation has been ascribed to the natural low oxygen tension present in the stem cell native environment (29). Xia and colleagues (30) explored the efficacy of adipose derived MSC secretome on gastric mucosal injury in a rodent model. The authors compared the effects of hypoxic $\left(5 \% \mathrm{O}_{2}\right)$ and normoxic $\left(21 \% \mathrm{O}_{2}\right)$-induced secretome and revealed improved cell migration, proliferation, viability, and angiogenesis in vitro that was more prominent with hypoxic-induced conditioned media.

There are some studies that report the use of a spheroid culture model as opposed to a monolayer culture. Spheroid cultures have been demonstrated to yield higher protein concentration than monolayer stem cell cultures and are thought to create a cytokine profile that is more physiologically relevant $(31,32)$. Redondo-Castro and colleagues (33) compared secretomes of human MSCs cultured either in a two-dimensional (2D) monolayer or a three-dimensional (3D) spheroid under interleukin-1 (IL-1) primed and unprimed conditions. The study revealed an increased secretion of factors such as IL-1 receptor antagonist, vascular endothelial growth factor, and granulocyte colony stimulating factors in the 3D spheroid culture model when compared to the 2D monolayer culture model.

The majority of studies utilize FBS in basal media due to the low amounts of antibodies it contains (32). However, concerns exist as FBS presents with high variability in composition and may contain animal contaminants, the latter of which may pose a risk for human recipients upon clinical translation. Consequentially, alternatives to FBS are increasingly being used and explored. These include human platelet lysates and various proprietary chemically defined serum-free media (SFM) (34), of which each have their advantages and disadvantages. For example, human platelet lysates have been reported to reduce immunological reactions and enhance stem-cell culture expansion. However, its use may be patient specific and has been reported to exhibit compromised immunosuppressive properties $(32,35)$. Furthermore, the use of SFM may be disadvantaged by a hefty price tag. To our knowledge, there are no studies that highlight the effect of FBS alternatives on the biomolecular profile of stem cell conditioned media.

Despite the regenerative potential of stem cell conditioned media, its widespread translation into clinical medicine is still precluded by lack of standardization in its formulary. Future research aimed at optimizing and standardizing stem cell conditioned media protocols specific to diseases are necessary.

\section{Characterizing content of stem cell conditioned media}

The clinical translatability of stem cell conditioned media is precluded by a paucity of protocols that identify bioactive molecules in stem cell conditioned media in a standardized and reproducible manner. Several methodologies exist that aim to accurately characterize contents in stem cell conditioned media. However, certain limitations, particularly those pertaining to test sensitivity, specificity, and reproducibility, persist (36).

Liquid chromatography-mass spectrometry (LC-MS) is one of the most common techniques that are used to characterize contents of stem cell conditioned media. LC-MS rely on n-terminal signal peptide found in secreted proteins, of which it aims to match such signal peptides into a library of previously identified proteins $(37,38)$. It is advantaged by its ability to analyze highly heterogenous compounds at low concentrations (39). 
One disadvantage of LC-MS is that the typical identification of proteins found in the secretome involves isolation of conditioned culture medium cells grown in serum-free medium (37). This growth in SFM has been reported to alter secretome proteomic profile $(27,28)$, and although secretome analysis could be done with serum containing conditioned media, this necessitates extensive fraction in the peptide level (40). Furthermore, the use of LC-MS hinges on the dearth of the protein library of which proteins found in the conditioned media can be compared. Finally, LC-MS are limited to the characterization of protein biomolecules in the secretome, thereby excluding analysis of other important metabolites and paracrine factors. These include mRNA, miRNA, and cellular organelles which have been well described as critical factors in the effectiveness of the stem cell secretome (41-43).

Studies exists that explored the sensitivity of LC-MS in protein identification in stem cell secretome. Nakashima et al. (44) conducted a proteomic analysis of human adipose-derived MSC secretome using LC-MS. The authors announced the identification of 128 proteins with this technique. Furthermore, the authors reported various proteins associated with growth, immune system processes, and reproduction, and concluded that LC-MS is useful for investigating new factors associated with cellular components, biological processes, and molecular functions in stem cell secretome. Nakashima and colleagues (44) utilized LC-MS to assess the proteomic profile of human umbilical cord matrix derived MSC secretome under static conditions. Using this analysis, the authors were able to identify $~ 2,400$ proteins.

Protein microarrays is an emerging technology that provides a platform to characterize thousands of proteins in parallel and at a high throughput (45). Additionally, protein microarrays offer faster results than other mainstream proteomic strategies (46). Analytical and functional protein microarrays are two major classes used to describe the applications of protein microarrays. Analytical protein microarrays include the antibody array that employs two different antibodies (a capture antibody and a florescent tagged detection antibody) to detect varying levels of protein in conditioned media. However, a risk exists for cross-reactivity of antibody proteins which may lead to a high number of false positives (47). Functional protein microarrays provide the ability to characterize protein functionality at the proteome level. Furthermore, it enables the study of various biochemical and binding properties of proteins in the secretome (48).

Mintz et al. (49) utilized a cytokine array kit to characterize the cytokine profile of CD34+ stem cell conditioned media. The authors ascertained 32 factors that were significantly more abundant in the conditioned medium when compared to control. Nguyen et al. (50) explored the use of MSC conditioned media in a swine model for myocardial infarction and reported improved cardiac parameters. The authors utilized protein array analysis to identify various angiogenic, antiapoptotic, and anti-remodeling factors. Other less commonly used methods for proteome analysis include ELISA, western blot analysis, and immunohistochemistry (51). However, these modalities are less sensitive than LC-MS and protein microarrays, and as such are often used as a confirmatory analysis (51).

Non-proteomic analyses have also been employed to characterize the non-protein components in stem cell secretome. Most important include assays that identify miRNAs. MiRNAs are an emerging class of non-coding RNA that regulate gene expression via their interaction with mRNA targets (52). There is increasing evidence that supports the role in various types of miRNA's in stem cell regenerative potential (53-55). Nucleotide based microarrays are the most commonly used method to identify miRNA in stem cell secretomes. Innovation in bioinformatics and improvements on search algorithms allow researchers to match nucleotide sequencings with an online database (56).

Ferguson et al. (57) utilized a nucleotide-based microarray to profile specific miRNAs found in MSC secretomes. The authors merged the results of their analysis with network bioinformatics to isolate the dominant biological processes and pathways modulated by their identified miRNAs. They then used the MSC-derived secretome to assess its proliferative and antiapoptotic effects on cardiomyocytes and cardiac fibroblasts. The authors revealed a reduction in apoptosis of cardiomyocytes and reduced collagen production by cardiac fibroblasts all of which were linked the pathways ascertained by network analysis.

There is great potential in utilizing emerging technologies and technological updates, such as bioinformatics, LC-MS, and tissue array kits, to develop standardized protocols for analyzing content in stem cell secretome. Clinical translation of stem cell conditioned media will partially hinge on modalities that accurately characterize the biomolecular content of stem cell secretomes in an accurate, efficient, and reproducible manner. 


\section{Dosing and delivery}

Safety and efficacy profiles are necessary for the widespread use of stem cell secretome as an effective therapeutic. As such, protocols that ascertain dosages, elucidate pharmacokinetics, and formulate in vivo modes of delivery in a disease specific manner are critical. This is vital for the therapeutic indexing of stem cell secretome in regenerative medicine (58). It is important to note that while stem cell secretomes may harbor biomolecular material that is conducive to tissue regeneration, there still remains a theoretical risk of immunogenicity due to evidence that MHC molecules may be transferred via extracellular vesicles (59). This risk for immunogenic response may be increased with a higher dose of stem-cell secretome, thereby emphasizing the need to characterize and dose stem cell conditioned media. Furthermore, the biomolecular milieu found in stem cell secretomes may have a deleterious effect at higher doses. Venugopal et al. (60) explored the neuroprotective effects of rat BMSC secretome in an in vitro model of excitotoxicity. Specifically, the authors assessed the effects of various passages (passage 3, 5, and 8) and doses $(0.05,0.1,0.5$, and $1 \mu \mathrm{g} / \mathrm{mL})$ of rat BMSC secretome in preventing neurodegeneration of a hippocampal cell line treated with kainic acid. The authors revealed greater protection against kainic acid induced cell apoptosis and necroptosis by earlier passage cell lines (passage 3 and 5) treated at lower doses $(0.05$ and $0.1 \mu \mathrm{g} / \mathrm{mL})$. Furthermore, the authors demonstrated improved antioxidant activity of low dose BMSC $(0.05$ and $0.1 \mu \mathrm{g} / \mathrm{mL})$ when compared to higher doses. The authors concluded that early passaged (passage 3 and 5) and low dose (0.05 and $0.1 \mu \mathrm{g} / \mathrm{mL})$ BMSC exerted neuroprotective properties, whereas higher doses from later passages were detrimental to neurons. To our knowledge, no studies exist that calculate the therapeutic window of various stem cell secretome formularies.

Stem cell secretome harbor varying kinetic and degradation properties, particularly in vivo. For example, there is significant heterogeneity reported for the halflife of miRNA in mammalian cells, with many isoforms demonstrating longer half-lives ( $>24$ hours), while others presenting a half-life ranging from 4 to 14 hours (61). Similarly, protein stability may vary in conditioned media. Attempts to inject stem cell secretome into blood circulation have been discouraging, as evidence reveals rapid uptake and clearance by macrophages in the reticuloendothelial system $(62,63)$. As such, there have been various modalities employed to prolong stem cell secretome half-life and duration of effect in vivo.

Hydrogel systems is one modality that has shown significant promise. Hydrogels are $3 \mathrm{D}$ crosslinked hydrophilic polymers that form matrices with high water content. These polymers are from natural or synthetic origins and have similarities to the native extracellular matrix (64). The strength of its applicability is its ability to be tuned to customize its degradation rate, thereby providing researchers the ability to modulate secretome release. Various methods exist for incorporating stem cell secretomes into hydrogel. One method includes mixing stem cell secretomes with hydrogel polymers before the addition of crosslinkers to gel the composite (54). Alternatively, stem cell secretome can be physically integrated into a hydrogel matrix after crosslinking. This technique requires that the hydrogel be dehydrated to remove water, and subsequently soaked in a medium containing stem cell secretomes. However, this requires the hydrogel to have pores larger than the secretome. One technique involves mixing stem cell secretome with the polymers and crosslinkers simultaneously (65). Interestingly, Luo et al.'s research group (66) fabricated synthetic analogues for stem cells as a therapeutic modality for acute myocardial infarction in mice. The authors utilized packaged secreted factors from human bone marrow-derived MSCs into poly (lactic-coglycolic acid) microparticles and subsequently coated them with MSC membranes. The authors revealed structural stability upon cryopreservation and lyophilization of the synthetic stem cell analogue.

Waters et al. (67) explored the regenerative capability of human adipose-derived stem cell (ADSC) secretome, loaded in hydrogel, on injured cardiac tissue. The authors reported no inflammatory response at 14 days after injection hydrogel + secretome construct in a myocardial infarcted (MI) rat model. Furthermore, the authors reported improved cardiac function parameters at day seven in their in vivo MI rat model in the cohort who received an injection of hydrogel + secretome when compared to control and secretome alone. The authors concluded that the secretome loaded hydrogel composite was able to improve heart function after the characteristic initial decline caused by acute MI cardiac remodeling. Han et al. (68) utilized human umbilical cord MSC-derived secretome encapsulated in hydrogel to promote cardiac repair. Using an animal in vivo MI model, the authors revealed improved cardiac function at 21 days after MI for the hydrogel coated secretome group when compared to control. Furthermore, the authors confirmed steady releases of stem cell secretome in the hydrogel construct over 22 days. The authors concluded that 
human umbilical cord MSC-derived secretome in a hydrogel construct was effective in improving cardiac function after MI.

Significant advancements focused on identifying safe dosage level and generating optimal modes of delivery are necessary for the translation of stem cell secretome as a therapeutic. While hydrogel has shown significant progress as a vehicle construct for secretome, there still lacks disease specific dosing parameters for stem cell secretome, thereby precluding research on safety parameters.

\section{Mechanisms of action}

There is a dearth of evidence that establishes the regenerative capabilities of stem cell conditioned media. Multiple biomolecular pathways have been implicated, most of which can be categorized into the following mechanisms: (I) direct stimulation of cell proliferation and migration through activation of various cell proliferative pathways; (II) immunomodulation to facilitate healing (69); (III) stabilization of redox-microenvironment; and (IV) direct anti-apoptotic and anti-senescent effect mediated by stem cell secretome.

Park et al. (70) explored the effect of human adipose derived MSC secretome on stimulating the proliferative and migratory capacity of various skin cell types. The authors reported on the secretome's capability to exert significant mitogenic effects through the activation of PI3K/Akt and/or FAK/ERK1/2 signaling pathways. Furthermore, their results from a scratch wound assay demonstrated significant motogenic effects of the secretome on skin cells. Martins et al. (71) assessed AFS-CM's capability to stimulate axonal growth of Wistar rat cortical and hippocampal neurons. The authors demonstrated a $132.5 \%$ and $130.3 \%$ increase in axon growth of cortical and hippocampal axons respectively $(\mathrm{P}<0.001)$. Further analysis revealed that depletion of BDNGF from the secretome prevented axonal growth, thus implicating BDGNF as being responsible for the seen effect through the activation ERK and PI3K-Akt pathway. Guo et al. (72) tested the in vitro efficacy of AFS secretome on Schwann cell viability and proliferation. The authors reported increased Schwann cell proliferation and viability of AFS-secretome treated cells at 24 and 48 hours when compared to controls (72). Furthermore, the authors revealed increased neurite growth of rat dorsal root ganglion for groups treated with the secretome when compared to control. Xin et al. (55) assessed the efficacy of systemic MSC secretome therapy on the functional recovery and neuroplasticity after stroke in adult male Wistar rats. In their study the authors subjected 12 mice to 2 hours of middle cerebral artery occlusion, followed by tail injection of $100 \mu \mathrm{g}$ of protein from MSC secretome (treatment group, $n=6$ ), or an equal volume of phosphate buffered saline (PBS). The authors report a statistically significant increase in neurite remodeling, neurogenesis, and angiogenesis along the ischemic boundary zone when compared among the treatment group when compared to control. The authors concluded that intravenous administration of cell-free MSC-generated exosomes post stroke improves functional recovery and enhances neurite remodeling, neurogenesis, and angiogenesis and represents a novel treatment for stroke.

The immunomodulatory capability of stem cell secretome has been identified as partially responsible for its regenerative effect. Kay and colleagues (73) published on MSC-conditioned media's ability to reduce severity and immune response in inflammatory arthritis. Using a murine inflammatory arthritis model, the authors reported lower levels of leukocyte accumulation in the joint synovium of MSC-conditioned media groups when compared to control at day 3 of post-arthritis induction $(\mathrm{P}<0.05)$. The authors also reported enhanced FOXP3 expression (a key transcriptional regulator for $\mathrm{T}$ regulatory cell development and function) in the spleens and lymph nodes in proximity to the arthritic joint. Chen et al. (74) explored the therapeutic effect of human MSC conditioned media, cultured in either MSC growth medium or neural regeneration laboratory medium, for both in vitro and in vivo models of spinal cord injury. The authors revealed lower levels of nitric oxide production for axons treated with lipopolysaccharide (LPS) and conditioned media, indicating an attenuated inflammatory response. Guo et al. (75) demonstrated the ability of ESC conditioned media to improve macrophage function in spinal cord injury. In their in vivo spinal cord injury model, the authors demonstrated the conditioned media's ability to enhances myelin debris clearance, rescue impaired phagocytic capacity from myelin impaired macrophages, enhances lipid efflux from myelin impaired macrophages, and reduce the formation of foamy cells around the cite of injury. Zhao et al. (76) explored the role of ADSCs on chondrogenic regeneration and subsequent amelioration of knee osteoarthritis. The authors reported that exosomes isolated from ADSCs, when co-cultured with activated synovial fibroblasts, downregulated the expression of pro-inflammatory markers IL-6, NF- $\kappa \mathrm{B}$ and tumor necrosis factor- $\alpha$, while they upregulated the expression of the anti-inflammatory cytokine IL-10. The opposite observations were made without exosomes. 
Some studies highlight stem cell secretome ability to stabilize cellular redox environment for an optimal environment conducive to regeneration. Hong et al. (77) utilized conditioned media derived from ADSCs and growth hypoxic (HCM) conditions, to explore its effect on hepatocytes in an in vitro model of toxic hepatic injury. The authors revealed that ADSC-CM downregulated intracellular reactive oxygen species $(\mathrm{P}<0.005)$ and upregulated mRNA and protein expression of superoxide dismutase, glutathione peroxidase, and catalase $(\mathrm{P}<0.05)$. These findings were also validated in their in vivo model of hepatic injury. Niu et al. (78) tested the capability of BMSC conditioned media to alleviate oxidative stress induced injury on neural stem cells, thereby recapitulating events that occur during spinal cord injury. The author's in vitro experiments revealed BMSC conditioned media's ability to improve survival of stem cells when exposed to $100 \mu \mathrm{M}$ of $\mathrm{H}_{2} \mathrm{O}_{2}$ in a dose dependent manner $(\mathrm{P}<0.001)$. Furthermore, the authors reported an increase in superoxide dismutase and decrease in malondialdehyde (a marker for oxidative stress induced cell injury) among neural stem cells treated with BMSC conditioned media when compared to control. Chen et al. (79) investigated the role of MSC conditioned media on attenuating irradiation induced damage of cardiac fibroblast cells. In their in vitro analysis, the authors discovered that MSC conditioned media + irradiated group had lower levels of malondialdehyde, and increased cellular protein levels of catalase, superoxide dismutase, and glutathione peroxidase when compared the irradiated group.

Multiple studies have reported on the anti-apoptotic and anti-senescent effects of stem cell secretome. Shologu and colleagues (80) reported MSC secretome to be effective in mitigating hypoxia-induced pulmonary epithelial injury. Specifically, the authors reported that rat alveolar epithelial cells that were pre-treated with either BMSC or ADSC secretome demonstrated increased cell survival when compared to control $(\mathrm{P}<0.001)$. Furthermore, the authors reported that MSC secretome facilitated p38 mitogen-activated protein kinase expression. Cells pretreated with BMSC secretome had increased nuclear translocation of Bcl-2 $(\mathrm{P}<0.001)$, whereas ADSC secretome significantly increased cytoplasmic $(\mathrm{P}<0.001)$ and nuclear $(\mathrm{P}<0.001)$ translocation of Bcl-2 when compared to hypoxic control. Similarly, Xu et al. (81) reported on MSC-CM's direct anti-apoptotic ability in $\mathrm{H}_{2} \mathrm{O}_{2}$ treated hepatocytes. The authors discovered MSC-CM's ability to increase cellular $\mathrm{Bcl}-2 / \mathrm{Bax}$ ratio when compared to control $(\mathrm{P}<0.05)$. Furthermore, the authors revealed that MSC-CM was able to downregulate miR143, a miRNA which the authors found to play a role in cell cycle arrest. Wang et al. (82) published their findings of AFS-CM's role in reducing replicative senescence of human MSCs. In their study, the authors reported a reduction in S-beta-galactosidase expression among human MSCs treated with AFS-CM when compared to control. Furthermore, the authors reported that AFS-CM downregulated senescence associated genes p16 and p53, despite an upregulation of $\mathrm{p} 21$. Zhu et al. (83) demonstrated the ability of embryonic MSC secretome to prevent $\mathrm{H}_{2} \mathrm{O}_{2}$-induced expression of cellular senescence gene p21 in cardiomyocytes. They also demonstrated lower $\mathrm{H}_{2} \mathrm{O}_{2}$-induced $\beta$-gal-activity among cardiomyocytes pre-treated with embryonic MSC exosome. The authors were able to isolate this protective effect to the lncRNA MALAT1 (metastasis-associated lung adenocarcinoma transcript 1) signaling pathway, which when activated led to a reduction in $N F-\kappa B$ activity and TNF- $\alpha$. The authors concluded that the protective effects of embryonic MSCs were due to activation of the lncRNA MALAT1/NF- $\kappa \mathrm{B} / \mathrm{TNF}-\alpha$ pathway.

The elucidation of mechanisms of action is critical for the development, standardization, and translation of stem cell secretome as a therapeutic agent for care providers. Multiple molecular pathways have been postulated to play a role in the regenerative processes of stem cell secretome. However, the majority of the literature all hint towards stem cells secretome's ability to stimulate cellular proliferation and migration, modulate host immune response, stabilize redox microenvironment, and mitigate stress induced cell apoptosis and senescence.

\section{Conclusions}

There has been an increasing number of investigations focused on harnessing the power of stem cells to facilitate tissue regeneration and recovery. Insights into mechanisms of action have revealed that it's the stem cell paracrine activity, packaged in the secretome, and not cell engraftment and differentiation, as defining the therapeutic ability of stem cell secretome. Despite multiple in vitro, in vivo, and clinical studies highlighting its therapeutic effectiveness, areas of concerns remain that precludes its clinical translational. Future studies should aim at uncovering best modes of stem cell conditioned media formulation, and analysis.

\section{Acknowledgments}

Funding: None. 


\section{Footnote}

Reporting Checklist: The authors have completed the Narrative Review reporting checklist. Available at http:// dx.doi.org/10.21037/atm-20-5030

Conflicts of Interest: All authors have completed the ICMJE uniform disclosure form (available at http://dx.doi. org/10.21037/atm-20-5030). The authors have no conflicts of interest to declare.

Ethical Statement: The authors are accountable for all aspects of the work in ensuring that questions related to the accuracy or integrity of any part of the work are appropriately investigated and resolved. Ethical approval was not required for this study design.

Open Access Statement: This is an Open Access article distributed in accordance with the Creative Commons Attribution-NonCommercial-NoDerivs 4.0 International License (CC BY-NC-ND 4.0), which permits the noncommercial replication and distribution of the article with the strict proviso that no changes or edits are made and the original work is properly cited (including links to both the formal publication through the relevant DOI and the license). See: https://creativecommons.org/licenses/by-nc-nd/4.0/.

\section{References}

1. Talman V, Ruskoaho H. Cardiac fibrosis in myocardial infarction--from repair and remodeling to regeneration. Cell Tissue Res 2016;365:563-81.

2. Bradbury EJ, Burnside ER. Moving beyond the glial scar for spinal cord repair. Nat Commun 2019;10:3879.

3. Yun MH. Changes in regenerative capacity through lifespan. Int J Mol Sci 2015;16:25392-432.

4. $\mathrm{Ng} \mathrm{M}$, Song S, Piuzzi NS, et al. Stem cell industry update: 2012 to 2016 reveals accelerated investment, but market capitalization and earnings lag. Cytotherapy 2017;19:1131-9.

5. Delanois RE, Etcheson JI, Sodhi N, et al. Biologic therapies for the treatment of knee osteoarthritis. J Arthroplasty 2019;34:801-13.

6. Rajabzadeh N, Fathi E, Farahzadi R. Stem cell-based regenerative medicine. Stem Cell Investig 2019;6:19.

7. Mahla RS. Stem cells applications in regenerative medicine and disease therapeutics. Int J Cell Biol 2016;2016:6940283.

8. Noguchi H, Miyagi-Shiohira C, Nakashima Y, et al.
Induction of expandable tissue-specific progenitor cells from human pancreatic tissue through transient expression of defined factors. Mol Ther Methods Clin Dev 2019;13:243-52.

9. Gorecka J, Kostiuk V, Fereydooni A, et al. The potential and limitations of induced pluripotent stem cells to achieve wound healing. Stem Cell Res Ther 2019;10:87.

10. Takahashi K, Tanabe K, Ohnuki M, et al. Induction of pluripotent stem cells from adult human fibroblasts by defined factors. Cell 2007;131:861-72.

11. Takahashi K, Yamanaka S. Induction of pluripotent stem cells from mouse embryonic and adult fibroblast cultures by defined factors. Cell 2006;126:663-76.

12. Yu J, Vodyanik MA, Smuga-Otto K, et al. Induced pluripotent stem cell lines derived from human somatic cells. Science 2007;318:1917-20.

13. Prochazkova M, Chavez MG, Prochazka J, et al. Embryonic versus adult stem cells. Stem Cell Biol Tissue Eng Dent Sci 2015;249-62.

14. Saeedi P, Halabian R, Imani Fooladi AA. A revealing review of mesenchymal stem cells therapy, clinical perspectives and modification strategies. Stem cell Investig 2019;6:34.

15. Tao H, Chen X, Wei A, et al. Comparison of teratoma formation between embryonic stem cells and parthenogenetic embryonic stem cells by molecular imaging. Stem Cells Int 2018;2018:7906531.

16. Cruz IB, Severo AL, Azzolin VF, et al. Regenerative potential of the cartilaginous tissue in mesenchymal stem cells: update, limitations, and challenges. Rev Bras Ortop 2016;52:2-10.

17. Gnecchi M, He H, Liang OD, et al. Paracrine action accounts for marked protection of ischemic heart by Aktmodified mesenchymal stem cells. Nat Med 2005;11:367-8.

18. Teixeira FG, Carvalho MM, Sousa N, et al. Mesenchymal stem cells secretome: a new paradigm for central nervous system regeneration? Cell Mol Life Sci 2013;70:3871-82.

19. MEDLINE@: Description of the Database. [cited 2020 Nov 20]. Available online: https://www.nlm.nih.gov/bsd/ medline.html

20. Daneshmandi L, Shah S, Jafari T, et al. Emergence of the stem cell secretome in regenerative engineering. Trends in Biotechnology 2020;38:1373-84.

21. Hughes P, Marshall D, Reid Y, et al. The costs of using unauthenticated, over-passaged cell lines: how much more data do we need? Biotechniques 2007;43:575-86.

22. Gutbier S, May P, Berthelot S, et al. Major changes of cell function and toxicant sensitivity in cultured cells 
undergoing mild, quasi-natural genetic drift. Arch Toxicol 2018;92:3487-503.

23. Pawitan JA. Prospect of stem cell conditioned medium in regenerative medicine. Biomed Res Int 2014;2014:965849.

24. Sagaradze G, Grigorieva O, Nimiritsky P, et al. Conditioned medium from human mesenchymal stromal cells: towards the clinical translation. Int J Mol Sci 2019;20:1656.

25. Park BS, Kim WS, Choi JS, et al. Hair growth stimulated by conditioned medium of adipose-derived stem cells is enhanced by hypoxia: evidence of increased growth factor secretion. Biomed Res 2010;31:27-34.

26. Di Santo S, Yang Z, Wyler von Ballmoos M, et al. Novel cell-free strategy for therapeutic angiogenesis: in vitro generated conditioned medium can replace progenitor cell transplantation. PLoS One 2009;4:e5643.

27. Bakondi B, Shimada IS, Perry A, et al. CD133 identifies a human bone marrow stem/progenitor cell sub-population with a repertoire of secreted factors that protect against stroke. Mol Ther 2009;17:1938-47.

28. Chang CP, Chio CC, Cheong CU, et al. Hypoxic preconditioning enhances the therapeutic potential of the secretome from cultured human mesenchymal stem cells in experimental traumatic brain injury. Clin Sci (Lond) 2013;124:165-76.

29. Paquet J, Deschepper M, Moya A, et al. Oxygen tension regulates human mesenchymal stem cell paracrine functions. Stem Cells Transl Med 2015;4:809-21.

30. Xia X, Chiu PWY, Lam PK, et al. Secretome from hypoxia-conditioned adipose-derived mesenchymal stem cells promotes the healing of gastric mucosal injury in a rodent model. Biochim Biophys Acta Mol Basis Dis 2018;1864:178-88.

31. Cesarz Z, Tamama K. Spheroid culture of mesenchymal stem cells. Stem Cells Int 2016;2016:9176357.

32. Phelps J, Sanati-Nezhad A, Ungrin M, et al. Bioprocessing of mesenchymal stem cells and their derivatives: toward cell-free therapeutics. Stem Cells Int 2018;2018:9415367.

33. Redondo-Castro E, Cunningham CJ, Miller J, et al. Changes in the secretome of tri-dimensional spheroidcultured human mesenchymal stem cells in vitro by interleukin-1 priming. Stem Cell Res Ther 2018;9:11.

34. Pachler K, Lener T, Streif D, et al. A Good Manufacturing Practice-grade standard protocol for exclusively human mesenchymal stromal cell-derived extracellular vesicles. Cytotherapy 2017;19:458-72.

35. Oikonomopoulos A, van Deen WK, Manansala AR, et al. Optimization of human mesenchymal stem cell manufacturing: the effects of animal/xeno-free media. Sci Rep 2015;5:16570.

36. Tabb DL, Vega-Montoto L, Rudnick PA, et al. Repeatability and reproducibility in proteomic identifications by liquid chromatography-tandem mass spectrometry. J Proteome Res 2010;9:761-76.

37. Adhikari S, Chen L, Huang P, et al. Proteomic analysis of secreted proteins from cell microenvironment. New York: Humana Press, 2017:45-58.

38. Pang B, Zhu Y, Lu L, et al. The applications and features of liquid chromatography-mass spectrometry in the analysis of Traditional Chinese Medicine. Evid Based Complement Alternat Med 2016;2016:3837270.

39. Beccaria M, Cabooter D. Current developments in LC-MS for pharmaceutical analysis. Analyst 2020;145:1129-57.

40. Weng Y, Sui Z, Shan Y, et al. In-depth proteomic quantification of cell secretome in serum-containing conditioned medium. Anal Chem 2016;88:4971-8.

41. Mead B, Tomarev S. Bone marrow-derived mesenchymal stem cells-derived exosomes promote survival of retinal ganglion cells through mirna-dependent mechanisms. Stem Cells Transl Med 2017;6:1273-85.

42. Mitchell R, Mellows B, Sheard J, et al. Secretome of adipose-derived mesenchymal stem cells promotes skeletal muscle regeneration through synergistic action of extracellular vesicle cargo and soluble proteins. Stem Cell Res Ther 2019;10:116.

43. Valadi H, Ekström K, Bossios A, et al. Exosome-mediated transfer of mRNAs and microRNAs is a novel mechanism of genetic exchange between cells. Nat Cell Biol 2007;9:654-9.

44. Nakashima Y, Nahar S, Miyagi-Shiohira C, et al. A liquid chromatography with tandem mass spectrometry-based proteomic analysis of the proteins secreted by human adipose-derived mesenchymal stem cells. Cell Transplant 2018;27:1469.

45. Sutandy FXR, Qian J, Chen CS, et al. Overview of protein microarrays. Curr Protoc Protein Sci 2013; Chapter 27:Unit 27.1.

46. Chen Z, Dodig-Crnković T, Schwenk JM, et al. Current applications of antibody microarrays. Clin Proteomics 2018;15:7.

47. Poetz O, Ostendorp R, Brocks B, et al. Protein microarrays for antibody profiling: Specificity and affinity determination on a chip. Proteomics 2005;5:2402-11.

48. Venkatarame Gowda Saralamma V, Vetrivel P, Kim $\mathrm{SM}$, et al. Proteome profiling of membrane-free stem 


\section{Page 10 of 11}

cell components by nano-LS/MS analysis and its antiinflammatory activity. Evid Based Complement Alternat Med 2019;2019:4683272.

49. Mintz PJ, Huang KW, Reebye V, et al. Exploiting human CD34+ stem cell-conditioned medium for tissue repair. Mol Ther 2014;22:149-59.

50. Nguyen BK, Maltais S, Perrault LP, et al. Improved function and myocardial repair of infarcted heart by intracoronary injection of mesenchymal stem cell-derived growth factors. J Cardiovasc Transl Res 2010;3:547-58.

51. Tran C, Damaser MS. Stem cells as drug delivery methods: application of stem cell secretome for regeneration. Adv Drug Deliv Rev 2015;82-83:1-11.

52. Eleuteri S, Fierabracci A. Insights into the secretome of mesenchymal stem cells and its potential applications. Int J Mol Sci 2019;20:4597.

53. Nakamura Y, Miyaki S, Ishitobi H, et al. Mesenchymal-stem-cell-derived exosomes accelerate skeletal muscle regeneration. FEBS Lett 2015;589:1257-65.

54. Qin Y, Wang L, Gao Z, et al. Bone marrow stromal/stem cell-derived extracellular vesicles regulate osteoblast activity and differentiation in vitro and promote bone regeneration in vivo. Sci Rep 2016;6:21961.

55. Xin H, Li Y, Cui Y, et al. Systemic administration of exosomes released from mesenchymal stromal cells promote functional recovery and neurovascular plasticity after stroke in rats. J Cereb Blood Flow Metab 2013;33:1711-5.

56. Kozomara A, Griffiths-Jones S. miRBase: integrating microRNA annotation and deep-sequencing data. Nucleic Acids Res 2011;39:D152-7.

57. Ferguson SW, Wang J, Lee CJ, et al. The microRNA regulatory landscape of MSC-derived exosomes: a systems view. Sci Rep 2018;8:1419.

58. Li SC, Han YP, Dethlefs BA, et al. Therapeutic window, a critical developmental stage for stem cell therapies. Curr Stem Cell Res Ther 2010;5:287-93.

59. Liu Q, Rojas-Canales DM, Divito SJ, et al. Donor dendritic cell-derived exosomes promote allograft-targeting immune response. J Clin Invest 2016;126:2805-20.

60. Venugopal C, Shamir C, Senthilkumar S, et al. Dosage and passage dependent neuroprotective effects of exosomes derived from rat bone marrow mesenchymal stem cells: an in vitro analysis. Curr Gene Ther 2017;17:379-90.

61. Marzi MJ, Ghini F, Cerruti B, et al. Degradation dynamics of microRNAs revealed by a novel pulse-chase approach. Genome Res 2016;26:554-65.

62. Charoenviriyakul C, Takahashi Y, Morishita M, et al. Cell
Gwam et al. Clinical translation of stem cell secretome: a review

type-specific and common characteristics of exosomes derived from mouse cell lines: yield, physicochemical properties, and pharmacokinetics. Eur J Pharm Sci 2017;96:316-22.

63. Wiklander OPB, Nordin JZ, O'Loughlin A, et al. Extracellular vesicle in vivo biodistribution is determined by cell source, route of administration and targeting. J Extracell Vesicles 2015;4:26316.

64. Bahram M, Mohseni N, Moghtader M. An introduction to hydrogels and some recent applications, emerging concepts in analysis and applications of hydrogels. Sutapa Biswas Majee, IntechOpen, 2016. doi: 10.5772/64301. Available online: https://www.intechopen.com/books/emergingconcepts-in-analysis-and-applications-of-hydrogels/anintroduction-to-hydrogels-and-some-recent-applications

65. Wang C, Wang $M, X u T$, et al. Engineering bioactive self-healing antibacterial exosomes hydrogel for promoting chronic diabetic wound healing and complete skin regeneration. Theranostics 2019;9:65-76.

66. Luo L, Tang J, Nishi K, et al. Fabrication of synthetic mesenchymal stem cells for the treatment of acute myocardial infarction in mice. Circ Res 2017;120:1768-75.

67. Waters R, Alam P, Pacelli S, et al. Stem cell-inspired secretome-rich injectable hydrogel to repair injured cardiac tissue. Acta Biomater 2018;69:95-106.

68. Han C, Zhou J, Liang C, Liu B, et al. Human umbilical cord mesenchymal stem cell derived exosomes encapsulated in functional peptide hydrogels promote cardiac repair. Biomater Sci 2019;7:2920-33.

69. Weiss ARR, Dahlke MH. Immunomodulation by mesenchymal stem cells (MSCs): mechanisms of action of living, apoptotic, and dead MSCs. Front Immunol 2019;10:1191.

70. Park SR, Kim JW, Jun HS, et al. Stem cell secretome and its effect on cellular mechanisms relevant to wound healing. Mol Ther 2018;26:606-17.

71. Martins LF, Costa RO, Pedro JR, et al. Mesenchymal stem cells secretome-induced axonal outgrowth is mediated by BDNF. Sci Rep 2017;7:4153.

72. Guo ZY, Sun X, Xu XL, et al. Human umbilical cord mesenchymal stem cells promote peripheral nerve repair via paracrine mechanisms. Neural Regen Res 2015;10:651-8.

73. Kay AG, Long G, Tyler G, et al. Mesenchymal stem cell-conditioned medium reduces disease severity and immune responses in inflammatory arthritis. Sci Rep 2017;7:18019.

74. Chen YT, Tsai MJ, Hsieh N, et al. The superiority of conditioned medium derived from rapidly expanded 
mesenchymal stem cells for neural repair. Stem Cell Res Ther 2019;10:390.

75. Guo L, Rolfe AJ, Wang X, et al. Rescuing macrophage normal function in spinal cord injury with embryonic stem cell conditioned media. Mol Brain 2016;9:48.

76. Zhao C, Chen JY, Peng WM, et al. Exosomes from adipose-derived stem cells promote chondrogenesis and suppress inflammation by upregulating miR-145 and miR-221. Mol Med Rep 2020;21:1881-9.

77. Hong HE, Kim OH, Kwak BJ, et al. Antioxidant action of hypoxic conditioned media from adipose-derived stem cells in the hepatic injury of expressing higher reactive oxygen species. Ann Surg Treat Res 2019;97:159-67.

78. Niu Y, Xia X, Song P, et al. Bone mesenchymal stem cell-conditioned medium attenuates the effect of oxidative stress injury on NSCs by inhibiting the Notch1 signaling pathway. Cell Biol Int 2019;43:1267-75.

79. Chen ZY, Hu YY, Hu XF, et al. The conditioned medium of human mesenchymal stromal cells reduces irradiation-induced damage in cardiac fibroblast cells. J

Cite this article as: Gwam C, Mohammed N, Ma X. Stem cell secretome, regeneration, and clinical translation: a narrative review. Ann Transl Med 2021;9(1):70. doi: 10.21037/atm-205030
Radiat Res 2018;59:555-64.

80. Shologu N, Scully M, Laffey JG, et al. Human mesenchymal stem cell secretome from bone marrow or adipose-derived tissue sources for treatment of hypoxiainduced pulmonary epithelial injury. Int J Mol Sci 2018;19:2996.

81. Xu X, Li D, Li X, et al. Mesenchymal stem cell conditioned medium alleviates oxidative stress injury induced by hydrogen peroxide via regulating miR143 and its target protein in hepatocytes. BMC Immunol 2017;18:51.

82. Wang B, Lee WYW, Huang B, et al. Secretome of human fetal mesenchymal stem cell ameliorates replicative senescence. Stem Cells Dev 2016;25:1755-66.

83. Zhu B, Zhang L, Liang C, et al. Stem cell-derived exosomes prevent aging-induced cardiac dysfunction through a novel exosome/lncRNA MALAT1/NF-кB/ TNF- $\alpha$ signaling pathway. Oxid Med Cell Longev 2019;2019:9739258. 